\title{
Algorithms for computations of Loday algebras' invariants
}

\begin{abstract}
The paper is devoted to applications of some computer programs to study structural determination of Loday algebras. We present how these computer programs can be applied in computations of various invariants of Loday algebras and provide several computer programs in Maple to verify Loday algebras' identities, the isomorphisms between the algebras, as a special case, to describe the automorphism groups, centroids and derivations.
\end{abstract}

